\title{
Prevalence and significance of sleep disordered breathing in adolescent athletes
}

\author{
To the Editor:
}

Sudden cardiac death in a young athlete is a tragic event, and emerging data suggest that the leading finding associated with sudden cardiac death in athletes is autopsy-negative sudden unexplained death [1]. Sleep disordered breathing (SDB) can be arrhythmogenic and lead to sudden cardiac death [2,3]. While obstructive sleep apnoea is generally prevalent in obese, inactive and/or aged subjects [4], little is known about its incidence in young athletes and highly active young people. Consequently, the impact of SDB on cardiovascular health of young athletes has not been fully elucidated. Athletes who participate in collision sports such as rugby and American football tend to exhibit risk factors for SDB such as large neck circumference and higher body mass index [5]. In this context, we investigated the prevalence and significance of SDB in young competitive athletes with a view to advancing the research conducted on athlete health.

47 male freshman athletes on a rugby football team at the Nippon Sport Science University underwent comprehensive cardiorespiratory evaluations in 2015 and 2016. The institutional review board of Showa University Fujigaoka Rehabilitation Hospital approved the protocol. Written informed consent was obtained from all subjects. This study was performed on 42 of the athletes (mean \pm SD $18.6 \pm 0.5$ years old, experience $8.2 \pm 4.0$ years) who completed SDB evaluations using a WatchPAT-200 device (Itamar Medical Ltd., Caesarea, Israel), a validated surrogate for polysomnography [6]. 18 (43\%) of the subjects evaluated met the definition set for SDB: an apnoea-hypopnoea index (AHI) of $\geqslant 5$ events $\cdot \mathrm{h}^{-1}$ over a total sleeping time of more than $3 \mathrm{~h}$. This SDB prevalence exceeded that previously reported in middle-aged men in the US and European general population $[4,7]$. The severity of the SDB was mild $\left(5 \leqslant \mathrm{AHI}<15\right.$ events. $\left.\mathrm{h}^{-1}\right)$ in 16 of the athletes and moderate $\left(15 \leqslant \mathrm{AHI}<30\right.$ events $\left.\cdot \mathrm{h}^{-1}\right)$ in two athletes. None of the athletes exhibited severe SBD (AHI: $\geqslant 30$ events.h ${ }^{-1}$ ).

Bioelectrical impedance analysis was performed to determine body composition, and neck circumference was manually measured. In the comparison between the athletes with and without SDB, there were no significant differences in the field-position, body composition, neck circumference or Epworth Sleepiness Scale (table 1). According to the analyses of the WatchPAT data collected during sleep, AHI showed a significant inverse correlation with the minimum oxygen saturation $(r=-0.696, p<0.0001)$ and significant positive correlations with the oxygen desaturation index $(\mathrm{r}=0.889, \mathrm{p}<0.0001)$ and average heart rate $(\mathrm{r}=0.426, \mathrm{p}<0.01)$. Hence, the sleeping SDB athletes exhibited a significantly lower minimum oxygen saturation, and significantly higher oxygen desaturation index and average heart rate, compared with the sleeping athletes without SDB (table 1).

Echocardiography, cardiopulmonary exercise testing (CPET) [8], 24-h Holter monitoring and ophthalmologic examinations were performed to evaluate cardiovascular abnormalities. No congenital heart or valvular diseases or apparent cardiomyopathies were detected in the echocardiography, although adaptive cardiac changes were found. The rugby football athletes exhibited greater mean values for the following parameters compared with previously reported reference values for Japanese males in their twenties [9]: left ventricular end-diastolic volume per body surface area (BSA), left ventricular mass per BSA, left atrial volume index and right atrial area. The parameters for systolic

@ERSpublications

Sleep disordered breathing (SDB) was more prevalent in adolescent athletes than expected, and several potential warning signs related to autonomic nerve activity appeared in SDB athletes. SDB screening may prevent associated downstream risks in the future. http://ow.ly/GQqK30nGm8r

Cite this article as: Iso Y, Kitai $\mathrm{H}$, Kyuno E, et al. Prevalence and significance of sleep disordered breathing in adolescent athletes. ERJ Open Res 2019; 5: 00029-2019 [https://doi.org/10.1183/ 23120541.00029-2019]. 
TABLE 1 Demographics and cardiopulmonary evaluation of athletes with and without sleep disordered breathing (SDB)

\begin{tabular}{|c|c|c|c|c|}
\hline & All & Non-SDB & SDB & p-value \\
\hline Position played forwards:backs & $18: 24$ & $8: 16$ & $10: 8$ & 0.15 \\
\hline Height $\mathrm{cm}$ & $174.2 \pm 6.5$ & $173.8 \pm 5.9$ & $174.7 \pm 7.3$ & 0.67 \\
\hline Weight kg & $84.1 \pm 13.8$ & $84.0 \pm 11.8$ & $84.1 \pm 16.5$ & 0.99 \\
\hline Body mass index $\mathrm{kg} \cdot \mathrm{m}^{-2}$ & $27.7 \pm 4.0$ & $27.8 \pm 3.2$ & $27.5 \pm 5.0$ & 0.85 \\
\hline BSA $\mathrm{m}^{2}$ & $2.0 \pm 0.2$ & $2.0 \pm 0.2$ & $2.0 \pm 0.2$ & 0.99 \\
\hline Neck circumference $\mathrm{cm}$ & $42.1 \pm 2.5$ & $42.2 \pm 1.8$ & $42.0 \pm 3.2$ & 0.73 \\
\hline Muscle weight, trunk kg & $29.3 \pm 3.5$ & $29.3 \pm 3.4$ & $29.3 \pm 3.8$ & 0.98 \\
\hline Muscle weight, limb kg & $28.6 \pm 4.1$ & $28.8 \pm 3.8$ & $28.3 \pm 4.6$ & 0.70 \\
\hline$\%$ Body fat & $20.3 \pm 7.9$ & $20.9 \pm 7.6$ & $19.5 \pm 8.4$ & 0.59 \\
\hline Epworth sleepiness scale & $9.0 \pm 4.0$ & $8.3 \pm 3.3$ & $9.9 \pm 4.7$ & 0.19 \\
\hline \multicolumn{5}{|l|}{ WatchPAT data } \\
\hline $\mathrm{AHI}$ & $5.4 \pm 4.4$ & $2.5 \pm 1.3$ & $9.4 \pm 3.9$ & $<0.0001$ \\
\hline ODI & $2.1 \pm 2.2$ & $0.9 \pm 0.8$ & $3.6 \pm 2.5$ & $<0.0001$ \\
\hline Mean oxygen saturation \% & $96.5 \pm 0.6$ & $96.6 \pm 0.7$ & $96.4 \pm 0.5$ & 0.37 \\
\hline Minimum oxygen saturation \% & $90.8 \pm 3.6$ & $91.9 \pm 2.6$ & $89.2 \pm 4.2$ & 0.02 \\
\hline Mean heart rate beats $\cdot \mathrm{min}^{-1}$ & $54.3 \pm 5.2$ & $52.4 \pm 5.5$ & $56.7 \pm 3.6$ & $<0.01$ \\
\hline \multicolumn{5}{|l|}{ Echocardiography } \\
\hline LVEF \% & $65.5 \pm 4.7$ & $66.0 \pm 4.7$ & $64.9 \pm 4.9$ & 0.50 \\
\hline LVEDV $\mathrm{mL}$ & $152.2 \pm 26.1$ & $151.9 \pm 27.7$ & $152.6 \pm 24.7$ & 0.93 \\
\hline LVEDV/BSA & $75.2 \pm 10.3$ & $75.1 \pm 10.7$ & $75.3 \pm 10.2$ & 0.96 \\
\hline LVESV mL & $53.6 \pm 10.6$ & $52.1 \pm 9.9$ & $55.5 \pm 11.4$ & 0.30 \\
\hline LVESV/BSA & $26.6 \pm 5.0$ & $25.8 \pm 4.3$ & $27.5 \pm 5.8$ & 0.29 \\
\hline Stroke volume $\mathrm{mL}$ & $98.8 \pm 19.5$ & $100.1 \pm 21.6$ & $97.2 \pm 17.0$ & 0.65 \\
\hline Stroke volume/BSA & $48.7 \pm 7.7$ & $49.4 \pm 8.7$ & $47.8 \pm 6.3$ & 0.52 \\
\hline LVDd mm & $52.8 \pm 3.1$ & $53.1 \pm 2.6$ & $52.4 \pm 3.8$ & 0.48 \\
\hline LVDd/BSA & $26.1 \pm 1.9$ & $26.3 \pm 1.6$ & $26.0 \pm 2.2$ & 0.61 \\
\hline IVS mm & $10.0 \pm 0.8$ & $9.9 \pm 0.8$ & $10.2 \pm 0.9$ & 0.28 \\
\hline PWT mm & $9.8 \pm 0.8$ & $9.8 \pm 0.7$ & $9.9 \pm 0.8$ & 0.64 \\
\hline LVM g & $197.9 \pm 30.4$ & $197.7 \pm 25.4$ & $198.1 \pm 36.9$ & 0.96 \\
\hline LVMI & $98.1 \pm 13.5$ & $98.4 \pm 12.9$ & $97.7 \pm 14.7$ & 0.88 \\
\hline LAD mm & $36.2 \pm 4.0$ & $36.6 \pm 4.3$ & $35.6 \pm 3.6$ & 0.40 \\
\hline LAVI & $29.9 \pm 6.0$ & $30.6 \pm 6.3$ & $28.8 \pm 5.6$ & 0.35 \\
\hline Right atrial area $\mathrm{mm}^{2}$ & $19.2 \pm 3.1$ & $19.4 \pm 3.2$ & $18.9 \pm 3.0$ & 0.61 \\
\hline Aortic diameter mm & $30.5 \pm 2.3$ & $30.4 \pm 2.4$ & $30.6 \pm 2.1$ & 0.80 \\
\hline$E / e^{\prime}$ & $6.4 \pm 1.4$ & $6.3 \pm 1.6$ & $6.6 \pm 1.0$ & 0.48 \\
\hline \multicolumn{5}{|l|}{ CPET } \\
\hline Rest heart rate beats $\cdot \mathrm{min}^{-1}$ & $72.5 \pm 7.4$ & $70.4 \pm 7.2$ & $75.0 \pm 7.0$ & 0.05 \\
\hline Peak heart rate beats. $\mathrm{min}^{-1}$ & $164.9 \pm 16.7$ & $162.4 \pm 17.1$ & $167.8 \pm 16.1$ & 0.32 \\
\hline Rest SBP mmHg & $127.3 \pm 17.0$ & $128.5 \pm 17.7$ & $125.8 \pm 16.4$ & 0.63 \\
\hline Peak SBP mmHg & $199.7 \pm 23.0$ & $196.2 \pm 20.6$ & $203.8 \pm 25.4$ & 0.31 \\
\hline $\mathrm{RMR} \mathrm{mL} \cdot \mathrm{min}^{-1} \cdot \mathrm{kg}^{-1}$ & $4.1 \pm 0.6$ & $4.3 \pm 0.5$ & $4.0 \pm 0.6$ & 0.20 \\
\hline Peak oxygen uptake $\mathrm{mL} \cdot \mathrm{min}^{-1} \cdot \mathrm{kg}^{-1}$ & $36.9 \pm 6.9$ & $36.6 \pm 6.3$ & $37.2 \pm 7.7$ & 0.81 \\
\hline \multicolumn{5}{|l|}{ 24-h Holter ECG ${ }^{\#}$} \\
\hline Mean heart rate beats $\cdot \mathrm{min}^{-1}$ & $67.4 \pm 5.8$ & $66.3 \pm 5.5$ & $68.5 \pm 6.1$ & 0.24 \\
\hline Maximum heart rate beats $\cdot \mathrm{min}^{-1}$ & $135.3 \pm 13.8$ & $136.7 \pm 16.4$ & $133.2 \pm 9.0$ & 0.43 \\
\hline Minimum heart rate beats $\cdot \mathrm{min}^{-1}$ & $36.1 \pm 4.2$ & $36.3 \pm 3.9$ & $35.8 \pm 4.7$ & 0.71 \\
\hline \multicolumn{5}{|l|}{ PVCs on Holter } \\
\hline Counts n $(\%)$ & & & & 0.02 \\
\hline 0 & $21(51.2 \%)$ & $12(50 \%)$ & $9(52.9 \%)$ & \\
\hline $1-99$ & $16(39.0 \%)$ & $12(50 \%)$ & $4(23.5 \%)$ & \\
\hline$\geqslant 100$ & $4(9.8 \%)$ & $0(0 \%)$ & $4(23.5 \%)$ & \\
\hline Run n $(\%)$ & & & & 1.00 \\
\hline 2 run & $2(4.9 \%)$ & $1(4.2 \%)$ & $1(5.9 \%)$ & \\
\hline $3-4$ run & $1(2.4 \%)$ & $1(4.2 \%)$ & $0(0 \%)$ & \\
\hline
\end{tabular}

Data are presented as mean \pm SD, unless otherwise stated. Total subjects $=42,24$ in the non-SDB group and 18 in the SDB. BSA: body surface area; AHI: apnoea-hypopnoea index; ODI: oxygen desaturation index; LV: left ventricle; EF: ejection fraction; EDV: end-diastolic volume; ESV: end-systolic volume; Dd: end-diastolic diameter; LVM: left ventricular mass; LVMI: left ventricular mass index; IVS: end-diastolic interventricular septum thickness; PWT: end-diastolic posterior wall thickness; LAD: left atrial diameter; LAVI: left atrial volume index; E wave: early diastolic filling velocity: e': early diastolic velocity; CPET: cardiopulmonary exercise testing; SBP: systolic blood pressure; RMR: resting metabolic rate; PVC: premature ventricular complex. ${ }^{\#}$ : number of SDB athletes was 17 because one athlete in the SDB group had a recording error. 
and diastolic function, by contrast, were normal in the athletes. There were no differences in the echocardiographic parameters between the SDB athletes and non-SDB athletes (table 1). The resting heart rate at the beginning of the CPET examination was higher in the SDB athletes than in the non-SDB athletes. There were no differences between the two groups in the systolic blood pressure at rest and at maximal exercise. Likewise, there were no significant differences between them in terms of peak oxygen consumption evaluated by respiratory gas analysis. The prevalence of subjects with premature ventricular complex (PVC) counts $>100$ per $24 \mathrm{~h}$ in ambulatory Holter recording was significantly greater in the SDB group than in the non-SDB group $(23.5 \%$ versus $0 \%, \mathrm{p}<0.05)$. According to reports from the team physicians none of the athletes suffered significant or fatal cardiac events during the 1-year follow-up.

Funduscopy and intraocular pressure measurements were performed by ophthalmologists who had no knowledge of the data from the cardiovascular evaluations. No microvascular damage or atherosclerotic changes in the retina were detected in the SDB athletes or non-SDB athletes, and no difference in intraocular pressure was observed between the two groups (mean \pm SD $13.0 \pm 0.5 \mathrm{mmHg}$ in the SDB athletes versus $14.2 \pm 0.5 \mathrm{mmHg}$ in the non-SDB athletes). The ophthalmologic examinations were repeated when the athletes became sophomores. None of the SDB or non-SDB athletes showed microvascular damage or atherosclerotic changes of the retina in this second round of examinations. However, the changes in intraocular pressure were slightly, but significantly, higher in the SDB athletes than in the non-SDB athletes $(1.04 \pm 0.14$ in the SDB versus $0.93 \pm 0.14$ in the non-SDB), although the pressures in the SDB athletes remained within normal range.

The subjects were given a questionnaire to identify incidences of concussion and sports-related injuries in the 1-year follow-up performed when the freshman became sophomores. No difference in the incidence of concussion was found between the two groups (27.8\% in the SDB athletes versus $34.8 \%$ in the non-SDB athletes), whereas the SDB group had a three-fold higher prevalence of athletes who underwent orthopaedic surgery for sports-related trauma or dropped out of the sport because of severe injury compared with the non-SDB group (28.8\% versus $8.7 \%)$. This difference in prevalence did not reach statistical significance. SDB affects neurocognitive function in children [10]. We thus surmise that poor attention in the SDB athletes could cause, at least in part, serious sport-related injury.

The results of the present study demonstrated that the heart rate in sleep, PVC counts in the Holter monitoring, and change ratio of the intraocular pressure were significantly higher in the teenage rugby players with SDB than in the non-SDB athletes. While no serious pathological conditions had yet developed in the young SDB athletes, SDB, even in the mild stage, is known to disturb the balance between the sympathetic and parasympathetic nerve activity in ways that potentially lead to adverse cardiovascular complications [11, 12]. The Wisconsin Cohort Study [13] found a significantly increased risk of combined coronary heart disease and heart failure in patients with mild SDB compared with the non-SDB group. SDB has also been shown to be associated with ophthalmic diseases including retinal vascular disease and glaucoma [14]. Thus, the present results would underscore the importance of SDB screening in young collision athletes, although further studies will be necessary to explore the mechanisms underlying onset of SDB in such athletes.

The limitations of this study were noteworthy. SDB assessment was performed on a single night, which may have introduced additional variability [15], even though it remains a standard. Also, the small number of subjects and the additional stratification of the study cohort by AHI limited the power of the study and introduced the possibility of type II error.

In conclusion, SDB was more prevalent in the adolescent rugby football athletes than expected, and several potential warning signs related to autonomic nerve activity appeared in the SDB athletes. SDB screening may prevent associated downstream risks, such as cardiovascular consequences in the future. Moreover, screening may minimise the present risk for sports-related injuries.

Yoshitaka Iso ${ }^{1,2}$, Hitomi Kitai ${ }^{1,3}$, Etsushi Kyuno ${ }^{1,2}$, Fumiyoshi Tsunoda ${ }^{1,2}$, Naoya Nishinaka ${ }^{1}$, Masahiko Funato $^{1}$, Eiichi Nishimura ${ }^{4}$, Shuichi Akihiro ${ }^{5}$, Hiroyuki Tanuma ${ }^{6}$, Toru Yonechi ${ }^{6}$, Eiichi Geshi ${ }^{3}$, Takeyuki Sambe ${ }^{1}$ and Hiroshi Suzuki ${ }^{7}$

${ }^{1}$ Showa University Research Institute for Sport and Exercise Sciences, Yokohama, Japan. ${ }^{2}$ Division of Cardiology, Showa University Fujigaoka Rehabilitation Hospital, Yokohama, Japan. ${ }^{3}$ Showa University School of Nursing and Rehabilitation Sciences, Yokohama, Japan. ${ }^{4}$ Division of Ophthalmology, Showa University Fujigaoka Rehabilitation Hospital, Yokohama, Japan. ${ }^{5}$ Athletic Dept, Nippon Sport Science University, Yokohama, Japan. ${ }^{6}$ Faculty of Sport Science, Nippon Sport Science University, Yokohama, Japan. ${ }^{7}$ Division of Cardiology, Showa University Fujigaoka Hospital, Yokohama, Japan. 
Correspondence: Yoshitaka Iso, Showa University Research Institute for Sport and Exercise Sciences, 2-1-1 Fujigaoka, Yokohama City, Kanagawa 227-8518, Japan. E-mail: yiso@med.showa-u.ac.jp

Received: Jan 302019 | Accepted: Jan 312019

Conflict of interest: None declared.

\section{References}

1 Asif IM, Harmon KG. Incidence and etiology of sudden cardiac death: new updates for athletic departments. Sports Health 2017; 9: 268-279.

2 Rossi VA, Stradling JR, Kohler M. Effects of obstructive sleep apnoea on heart rhythm. Eur Respir J 2013; 41 : 1439-1451.

3 Gami AS, Olson EJ, Shen WK, et al. Obstructive sleep apnea and the risk of sudden cardiac death: a longitudinal study of 10,701 adults. J Am Coll Cardiol 2013; 62: 610-616.

4 Arnardottir ES, Bjornsdottir E, Olafsdottir KA, et al. Obstructive sleep apnoea in the general population: highly prevalent but minimal symptoms. Eur Respir J 2016; 47: 194-202.

5 Peck B, Renzi T, Peach H, et al. Examination of risk for sleep-disordered breathing among college football players. J Sport Rehabil 2019; 28: 126-132.

6 Yalamanchali S, Farajian V, Hamilton C, et al. Diagnosis of obstructive sleep apnea by peripheral arterial tonometry: meta-analysis. JAMA Otolaryngol Head Neck Surg 2013; 139: 1343-1350.

7 Jennum P, Riha RL. Epidemiology of sleep apnoea/hypopnoea syndrome and sleep-disordered breathing. Eur Respir J 2009; 33: 907-914.

8 Iso Y, Suzuki H, Kyuno E, et al. Therapeutic potential of cycling high-intensity interval training in patients with peripheral artery disease: A pilot study. Int J Cardiol Heart Vasc 2018; 18: 30-32.

9 Daimon $\mathrm{M}$, Watanabe $\mathrm{H}$, Abe $\mathrm{Y}$, et al. Normal values of echocardiographic parameters in relation to age in a healthy Japanese population: the JAMP study. Circ J 2008; 72: 1859-1866.

10 Owens JA. Neurocognitive and behavioral impact of sleep disordered breathing in children. Pediatr Pulmonol 2009; 44: 417-422.

11 Floras JS. Sleep apnea and cardiovascular disease: an enigmatic risk factor. Circ Res 2018; 122: 1741-1764.

12 Chowdhuri S, Quan SF, Almeida F, et al. An official American Thoracic Society research statement: impact of mild obstructive sleep apnea in adults. Am J Respir Crit Care Med 2016; 193: e37-e54.

13 Hla KM, Young T, Hagen EW, et al. Coronary heart disease incidence in sleep disordered breathing: the Wisconsin Sleep Cohort Study. Sleep 2015; 38: 677-684.

14 West SD, Turnbull C. Obstructive sleep apnoea. Eye (Lond) 2018; 32: 889-903.

15 Levendowski DJ, Zack N, Rao S, et al. Assessment of the test-retest reliability of laboratory polysomnography. Sleep Breath 2009; 13: 163-167. 